\title{
Pericarditis with anaemia as a herald syndrome in a fatal presentation of cardiac lymphoma
}

\author{
Aish Sinha, ${ }_{1}^{1}$ Timothy Davies, ${ }^{2}$ Ahmad Saif, ${ }^{2}$ Andrew Apps $^{3}$
}

${ }^{1}$ Department of Cardiology, King's College Hospital, London, UK

${ }^{2}$ Department of Cardiology, Wycombe General Hospital, High Wycombe, UK

${ }^{3}$ Department of Cardiology, John Radcliffe Hospital, Headley Way, Oxford, UK

\section{Correspondence to} Dr Andrew Apps,

drandyapps@googlemail.com

Accepted 3 February 2016
CrossMark

\footnotetext{
To cite: Sinha A, Davies T, Saif $A$, et al. BMJ Case Rep Published online: [please include Day Month Year] doi:10.1136/bcr-2015212810
}

\section{SUMMARY}

Primary cardiac lymphoma $(\mathrm{PCL})$ is rare, accounting for $2 \%$ of all primary cardiac malignancies. Diagnosis is sometimes slow due to the non-specific nature of symptoms, causing a delay to treatment with potentially curative anthracycline chemotherapy. We report an unusual presentation of primary cardiac lymphoma in an immunocompetent man presenting with subacute isolated right-sided heart failure with pericarditis on a background of chronic anaemia and constitutional upset. Echocardiography demonstrated a pericardial mass invading the right atrium and compressing the tricuspid annulus. Diffuse large B-cell lymphoma was diagnosed after biopsy. This case highlights the importance of early imaging and hospitalisation in pericarditis with high-risk features such as high inflammatory markers, myocardial involvement (with troponin elevation), fever, immunosuppression or evidence of heart failure. The differential and diagnostic pathway of an intracardiac mass, and the treatment and prognosis of $\mathrm{PCL}$, are discussed.

\section{BACKGROUND}

Pericarditis is a common clinical presentation. It can be idiopathic, or secondary to trauma, autoimmune aetiology, infection or uraemia. Similarly, iron deficiency anaemia is also a common finding in clinical practice and should prompt investigations to exclude malignancy in the older population. Such investigations are often limited to colonoscopy and gastroscopy. A complex constellation of clinical findings, such as pericarditis, iron deficiency anaemia and systemic symptoms, not resolving with initial management, should prompt the clinician to think of other, more unusual, pathologies. Primary cardiac lymphoma (PCL) is a rare form of cardiac neoplasm, accounting for only $2 \%$ of all cardiac tumours. ${ }^{1}$ Although usually presenting inconspicuously, with non-specific symptoms of fever and malaise, making it difficult to diagnose, symptoms due to compression by a tumour mass and catastrophic systemic embolisation can also occur.

An intracardiac mass identified at initial imaging (usually echocardiography or CT) is often the first clue to a diagnosis of PCL. Subsequent histological diagnosis via tissue biopsy allows treatment (which may include chemotherapy and/or surgical resection) to be planned. Timely intervention improves the outcome of PCL, which otherwise carries a poor prognosis.

\section{CASE PRESENTATION}

A 65-year-old man presented with a 5-month history of general malaise, dyspnoea, fever and anorexia. These symptoms were thought to be secondary to a new iron deficiency anaemia (iron $8.5 \mu \mathrm{mol} / \mathrm{L}$, transferrin $1.88 \mathrm{~g} / \mathrm{L}$, transferrin saturation 18\%), which had prompted gastroscopy and colonoscopy 2 months previously. These investigations were normal, therefore ruling out common gastrointestinal pathology. The patient now described a three-day history of sharp central chest pain with relief sitting upright, bilateral leg oedema, episodes of presyncope relating to standing and dyspnoea graded at New York Heart Association class III prompting cardiology referral. On examination, his blood pressure was $90 / 60$, his jugular venous pressure was raised and he had ankle oedema bilaterally. In addition, he had a pericardial rub. ECG demonstrated atrial fibrillation with ventricular rate $100 \mathrm{bpm}$ and chest radiograph was unremarkable. Blood work demonstrated an inflammatory response with elevated $\mathrm{C}$ reactive protein $(185 \mathrm{mg} / \mathrm{L})$ and an erythrocyte sedimentation rate of $73 \mathrm{~mm} / \mathrm{h}$. Troponin I was significantly raised at $0.85 \mu \mathrm{g} / \mathrm{L}$. Autoantibodies and immunoglobulins were within normal limits, and lactate dehydrogenase was mildly elevated at $313 \mathrm{U} / \mathrm{L}$.

Transthoracic echocardiography (TTE) demonstrated an intrapericardial mass compressing the tricuspid annulus, with invasion of the right atrium (figure 1). Right ventricular function was severely impaired, while left ventricular function was preserved. MRI characterised the intrapericardial mass as a large cardiac tumour $(10 \times 7 \mathrm{~cm}$; figure 2$)$

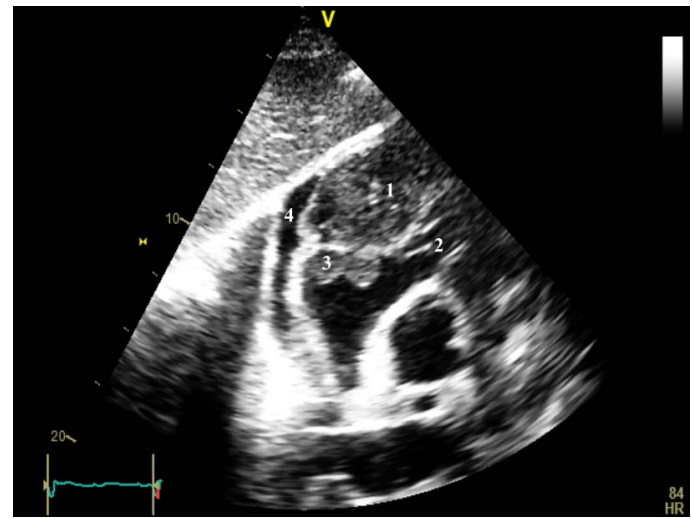

Figure 1 Echocardiogram (subcostal four-chamber view) demonstrating a large pericardial mass (1); compressing the right atrium and tricuspid valve annulus (2); with evidence of invasion of the right atrium (3). A small pericardial effusion is also noted (4). 


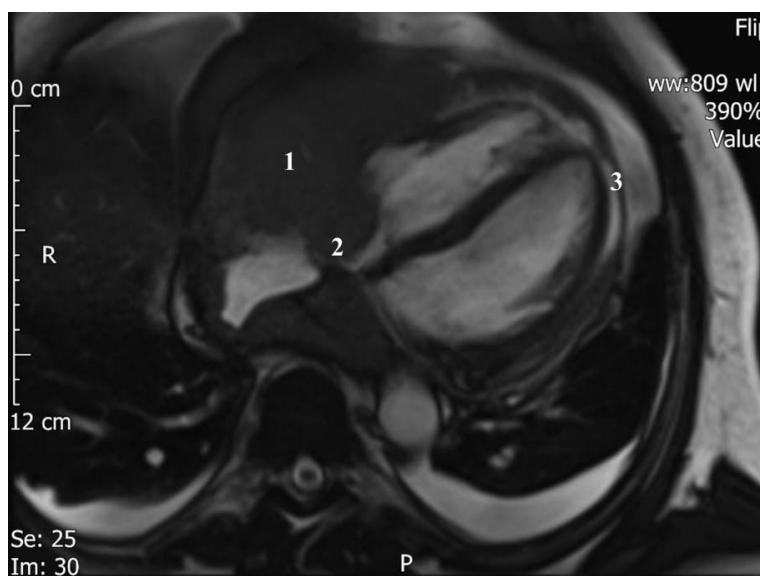

Figure 2 Steady-state free precession (SSFP) MRI scan demonstrating the large pericardial mass (1); compressing the right atrium and tricuspid valve annulus (2). The small pericardial effusion is also noted (3).

encasing the right ventricle up to the aortic arch and down to the inferior vena cava (video 1), with pedunculated masses seen in the right atrium and severe compression of the tricuspid valve (figure 2). The TTE (figure 1) and MRI (figure 2) both demonstrated a small pericardial effusion. Interestingly, the tumour extension also caused encasement of the right coronary artery (figure 3 and video 2). The patient was started on dexamethasone, with clinical improvement. Endomyocardial biopsy yielded high-grade lymphoid cells (figure 4), and immunohistochemical staining showed these cells were positive for CD20 and bcl-2 (figure 5), consistent with a diagnosis of diffuse large B-cell lymphoma. Unfortunately, the patient deteriorated due to

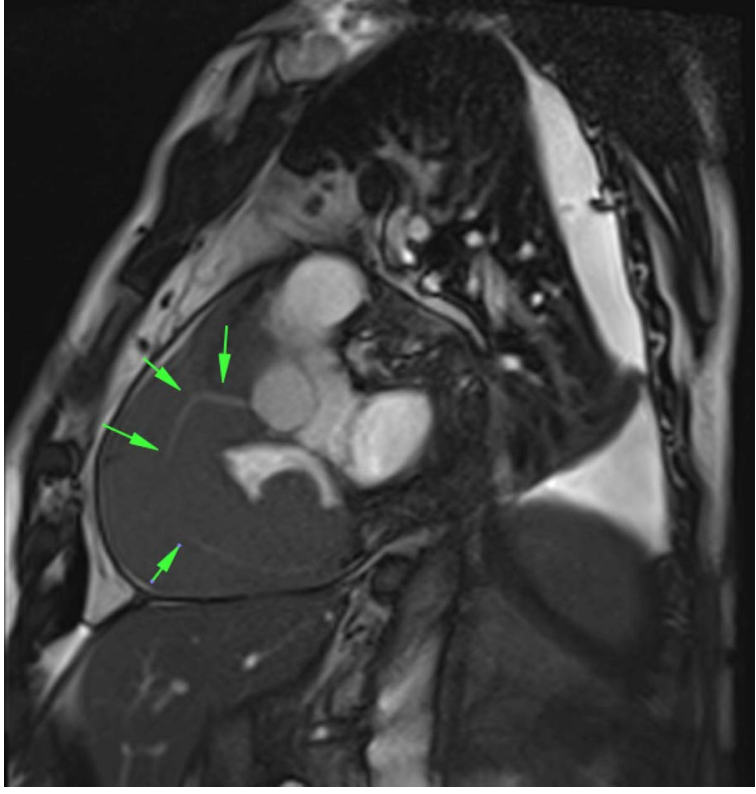

Figure 3 Single frame from short axis video at the atrioventricular valves showing encasement of the right coronary artery (green arrows).

right heart failure developing shock refractory to inotropic support, which proved fatal.

\section{DISCUSSION}

PCL is rare, accounting for $2 \%$ of all primary cardiac neoplasms. ${ }^{1}$ The commonest affected sites are the right atrium, right ventricle and the pericardium. ${ }^{2}$ Symptoms relate to tumour extension and/or embolism, with dyspnoea, chest pain

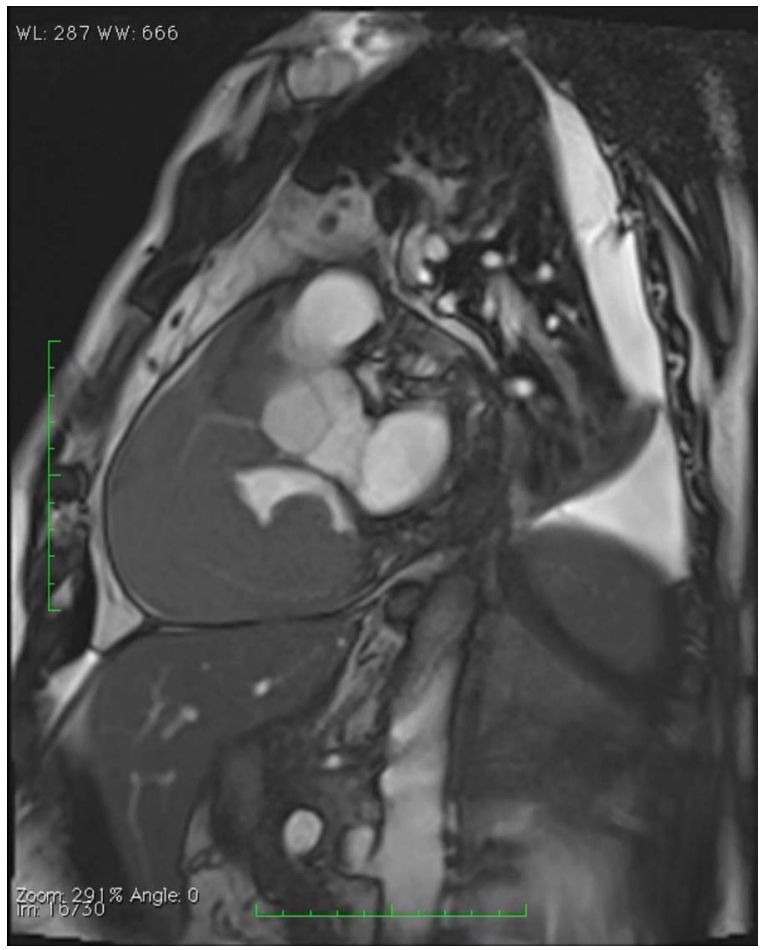

Video 2 Short axis video at the level of the atrioventricular valves showing tumour encasement of the right coronary artery and invasion of the right atrium.
Video 1 Short axis mid-left ventricle video showing tumour encasement of the right ventricle with diastolic dysfunction and delayed right ventricular filling. 


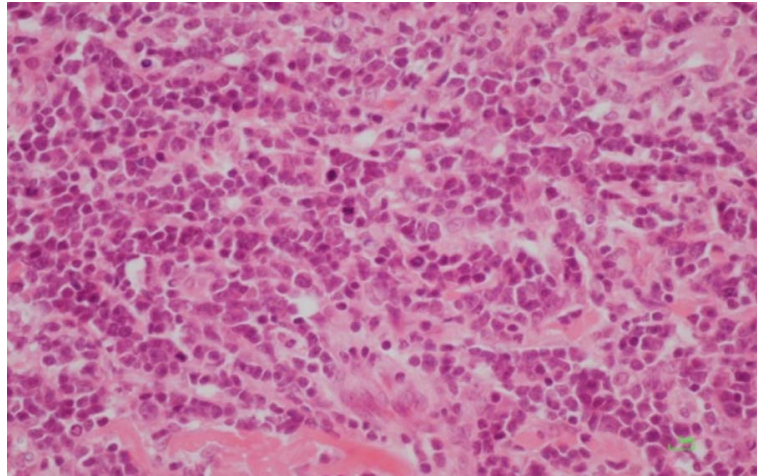

Figure 4 Section from the tumour mass demonstrating infiltration by sheets of medium to large sized lymphoid cells featuring round nuclei with small nucleoli and scanty amounts of cytoplasm. The H\&E $(x 40)$ stain confirms a high-grade lymphoma with high rates of mitosis and apoptosis.

and constitutional symptoms being the commonest presenting symptoms, occurring in $66 \%, 26 \%$ and $24 \%$ of patients, respectively. ${ }^{2}$ Anaemia is a common finding in lymphoproliferative disorders ${ }^{4}$ such as PCL, and the pathophysiology is thought to be related to abnormal iron metabolism. ${ }^{5}$ Our patient had progressive anaemia with no evidence of gastrointestinal blood loss; however, diagnosis was only made after chest pain developed. PCL usually presents after the fifth decade of life. ${ }^{6}$ B-cell lymphoma is the commonest type of PCL and is rapidly progressive, with median survival of less than 1 month without chemotherapy. ${ }^{7}$ Poor prognostic markers of PCL include left ventricular involvement, immunocompromise and the presence of extracardiac disease. ${ }^{7}$ In addition to these, anaemia itself is a poor prognostic marker of lymphoma for both overall and progression-free survival, ${ }^{4}$ therefore its early recognition alone may play a powerful role in reducing mortality.

Making a diagnosis of PCL is often slow, which is to some extent unavoidable given that symptoms such as fevers, anaemia and breathlessness will clearly not prompt echocardiography or thoracic CT prior to more basic investigations. However, with better access to advanced cardiac imaging when requested, the diagnosis is being made earlier with more patients being deemed suitable for receiving chemotherapy with associated survival extending to 30 months. $^{7}$ Surgical excision and radiotherapy offer less clear benefit, although selection bias may be at play, given that those presenting moribund are less likely to be

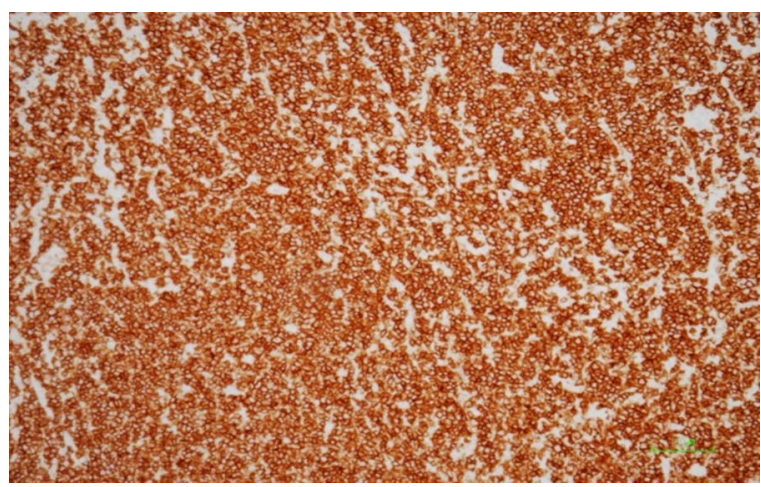

Figure 5 The sample stains positively for $\operatorname{CD} 20(\times 20)$, confirming the lymphoma to be a large B-cell lymphoma. offered chemotherapy. Our case is testament to the aggressive nature of this disease; despite timely histological diagnosis, the patient passed away within days.

Nearly all the published cases are first identified as right-sided masses at TTE, it thus seems sensible to postulate that early echocardiography affords the patient the best chance of being diagnosed when still a candidate for treatment. The difficulty here is that the denominator is huge. As discussed, cases commonly present with non-specific symptoms of breathlessness, fever, weight loss and lethargy_-symptoms that are often benign and investigated routinely as an outpatient. How does the physician pick out the needle from the haystack in deciding who needs early access to an echocardiogram? Rapidly progressive symptoms not responding to initial treatments, or, more critically, a constellation of symptoms (seen in this case as anaemia, chest pain, breathlessness and peripheral oedema) occurring sequentially, should prompt echocardiography to rule out a mass or vegetation.

The finding of an intracardiac mass has extensive differential diagnoses, including the more common primary cardiac neoplasms of myxoma (up to $50 \%$ of primary cardiac tumours), angiosarcoma, metastatic deposit and thrombus. ${ }^{8}$ Therefore, diagnosis of PCL requires further imaging with transoesophageal echocardiography, cardiac MRI, CT and positron emission tomography scanning, leading to endomyocardial biopsy. ${ }^{9}$ Only when this histological (or, more rarely, cytological via pericardial fluid) diagnosis is obtained, can the patient be considered for chemotherapy or surgery. Right-sided tumours are particularly difficult to debulk, however, and the role of surgery is limited, with the mainstay of treatment being an anthracycline-based chemotherapy regimen of cyclophosphamide, doxorubicin, vincristine and prednisolone (R-CHOP). ${ }^{10}$

We present an unusual case of PCL in an immunocompetent patient, who initially reported of a constellation of common clinical symptoms ascribed to a new iron deficiency anaemia. The case report highlights the importance of revisiting the extended differential diagnosis when regular diagnostic work up for common clinical presentations (in this case iron deficiency anaemia) yields no diagnosis. In addition, we highlight the importance of early imaging and hospitalisation in pericarditis with high-risk features, such as high inflammatory markers, myocardial involvement, fever and evidence of heart failure. $^{11}$

\section{Learning points}

- Pericarditis is idiopathic in $80-90 \%$ of cases, and can be safely managed as an outpatient. Features such as fever, high inflammatory markers, a subacute onset, immunosuppression, myocardial involvement (with troponin elevation) and heart failure, point to an increased risk of complications and warrant hospitalisation for early echocardiography and a full aetiological search. ${ }^{11}$

- The differential diagnoses of an intracardiac mass are extensive and include treatable causes-anticoagulation for thrombus, excision for myxoma and chemotherapy for lymphoma. The key is early diagnosis, requiring complex imaging and, often, endocardial biopsy.

- Primary cardiac lymphoma is rare and frequently fatal; however, if diagnosed early enough, it is a treatable and sometimes even curable disease. 
Acknowledgements The authors would like to thank our patient's wife for giving us consent to publish this case report. The authors would also like to thank Dr Wei Chuen Liong for his help in choosing the MRI images for this patient.

Contributors $A S, A h S$ and $A A$ were responsible for drafting of the manuscript for important intellectual content. AS, TD, AhS, AA were responsible for revision of the manuscript.

Competing interests None declared.

Patient consent Obtained.

Provenance and peer review Not commissioned; externally peer reviewed.

\section{REFERENCES}

1 Fernandes F, Soufen HN, lanni BM, et al. Primary neoplasms of the heart. Clinical and histological presentation of 50 cases. Arq Bras Cardiol 2001;76:231-7.

2 Burling F, Devlin G, Heald S. Primary cardiac lymphoma diagnosed with transesophageal endocardiography-guided endomyocardial biopsy. Circulation 2000;101:e179-81.
3 Nakata A, Hirota S, Takazakura E. Primary cardiac lymphoma diagnosed by percutaneous needle biopsy. Int J Cardiol 1998;65:201-3.

4 Moullet I, Salles G, Ketterer N, et al. Frequency and significance of anemia in non-Hodgkin's lymphoma patients. Ann Oncol 1998:9:1109-15.

5 Birgegård G. Managing anemia in lymphoma and multiple myeloma. Ther Clin Risk Manag 2008:4:527-39.

6 Hsueh SC, Chung MT, Fang R, et al. Primary cardiac lymphoma. J Chin Med Assoc 2006;69:169-74.

7 Petrich A, Cho SI, Billett H. Primary cardiac lymphoma: an analysis of presentation, treatment, and outcome patterns. Cancer 2011;117:581-9.

8 Lobo A, Lewis JF, Conti R. Intracardiac masses detected by echocardiography: case presentations and review of the literature. Clin Cardiol 2000;23:702-8.

9 Habertheuer $A$, Ehrlich $M$, Wiedemann $D$, et al. A rare case of primary cardiac B cell lymphoma. J Cardiothorac Surg 2014;9:14.

10 Armitage JO. My treatment approach to patients with diffuse large B-cell lymphoma. Mayo Clin Proc 2012;87:161-71.

11 Imazio M, Cecchi E, Demichelis B, et al. Indicators of poor prognosis of acute pericarditis. Circulation 2007;115:2739-44.

Copyright 2016 BMJ Publishing Group. All rights reserved. For permission to reuse any of this content visit http://group.bmj.com/group/rights-licensing/permissions.

BMJ Case Report Fellows may re-use this article for personal use and teaching without any further permission.

Become a Fellow of BMJ Case Reports today and you can:

- Submit as many cases as you like

- Enjoy fast sympathetic peer review and rapid publication of accepted articles

- Access all the published articles

- Re-use any of the published material for personal use and teaching without further permission

For information on Institutional Fellowships contact consortiasales@bmjgroup.com

Visit casereports.bmj.com for more articles like this and to become a Fellow 